\title{
DOCUMENTOS/
}

\section{Política Cultural de las \\ Regiones Autónomas del Caribe Nicaragüense Principales Lineamientos y Componentes del Plan de Desarrollo Cultural}

\begin{abstract}
Política Cultural de las Regiones Autónomas de la Costa Caribe de Nicaragua, respecto a los Pueblos Indígenas, Comunidades Afrodescendientes y Mestizos Costeños; así como de la Estrategia, Plan de Acción e Institucionalidad necesarias para su Implementación
\end{abstract}

Bilwi, Región Autónoma Atlántico Norte, 28 de Junio de 2012

\section{PRESENTACION}

Este documento presenta la política cultural de las Regiones Autónomas de la Costa Caribe de Nicaragua y sus principales lineamientos, en el marco de la definición y construcción, en esos ámbitos, de la institucionalidad autonómica para estas Regiones. Además incluye los principales componentes del Plan de Desarrollo Cultural, derivada de esos lineamientos.

Su definición ha sido posible luego de haberse concluido el proceso participativo que permitió obtener el Diagnóstico, Línea Base e Inventario Cultural de los Pueblos Indígenas, Comunidades Afro descendientes y Mestizos Costeños, de revisarse y analizarse el contenido y lineamientos de la Política Cultural que ha sido definida en el ámbito nacional.

Esta política cultural y sus lineamientos, al igual que de los componentes del plan de desarrollo cultural, cuenta con el aval de los Consejos Regionales Autónomos Norte y Atlántico Sur concluido el proceso de consulta con autoridades territoriales, sus comunidades y las autoridades de los órganos de la Administración Regional Autónoma, en ambas regiones.

La política cultural, aparte de su definición y contenido fundamental, y de sus lineamientos, incorpora los principios sobre los cuales se sustenta. Estos principios son un marco de orientación de la política nacional y regional, así como consideraciones de instrumentos internacionales sobre derechos de los Pueblos Indígenas y Afrodescendientes. Las bases de esta política está en el marco conceptual generada por los pueblos indígenas, comunidades afrodescendientes y mestizos costeños, el marco jurídico de la autonomía regional y la política cultural del Gobierno de Reconciliación y Unidad Nacional (GRUN).

\section{INTRODUCCIÓN}

Las Regiones Autónomas se caracterizan por contar con una amplia, profunda y rica diversidad cultural. La pluriculturalidad e interculturalidad son de las principales características que las diferencian del resto del país. La diversidad cultural, además se constituye en la fuerza que las potencia e impulsa la vida espiritual, afectiva, intelectual, social, política y económica de sus poblaciones multiétnicas.

Esta diversidad y riqueza cultural de las Regiones Autónomas, y su reconocimiento, en los ámbitos nacional e internacional, impulsa también, y se convierte en un medio que contribuye a garantizar la libertad, la armonía, la equidad y la justicia, la igualdad cultural, la cohesión social, el desarrollo sostenible y respetuoso, la gobernanza autonómica; $\mathrm{y}$, el ejercicio efectivo de los derechos de los pueblos indígenas y comunidades étnicas.

La riqueza cultural de los Pueblos Indígenas, Comunidades Afrodescendientes y Mestizos Costeños de estas Regiones, 
queda evidenciada mediante el Inventario y Mapeo de Recursos Culturales de los Pueblos Indígenas y Comunidades Afrodescendientes, y el mapeo de los Mestizos Costeños de la Costa Caribe de Nicaragua, a través del cual se identificó una primera lista que refleja 2,342 recursos culturales.

En el derecho internacional, el derecho a la cultura se refleja en el Convenio 169 de la OIT, de 1989, en la Declaración Universal de la UNESCO sobre la Diversidad Cultural del año 2001, en la Convención Sobre la Protección y Promoción de la Diversidad de las Expresiones Culturales del año 2005, y en la Declaración sobre los Derechos de los Pueblos Indígenas de las Naciones Unidas, del año 2007, entre otros instrumentos.

En el ámbito nacional, tal reconocimiento también se encuentra expresado en distintos instrumentos jurídicos, como la Constitución Política de Nicaragua, el Estatuto para la Autonomía de las Regiones Autónomas de la Costa Atlántica de Nicaragua (Ley No.28) y su Reglamento, entre otros.

En efecto, después de varios cambios en la Constitución de la República y con la promulgación de nuevas leyes, nuestro país ha avanzado en reconocerse constituida por diversos pueblos y hacia la conciencia de ser un Estado de naturaleza multiétnica, pluricultural y multilingüe, que debe vivir y desarrollarse bajo el principio de la interculturalidad ${ }^{1}$.

El Estatuto de la Autonomía de las Regiones

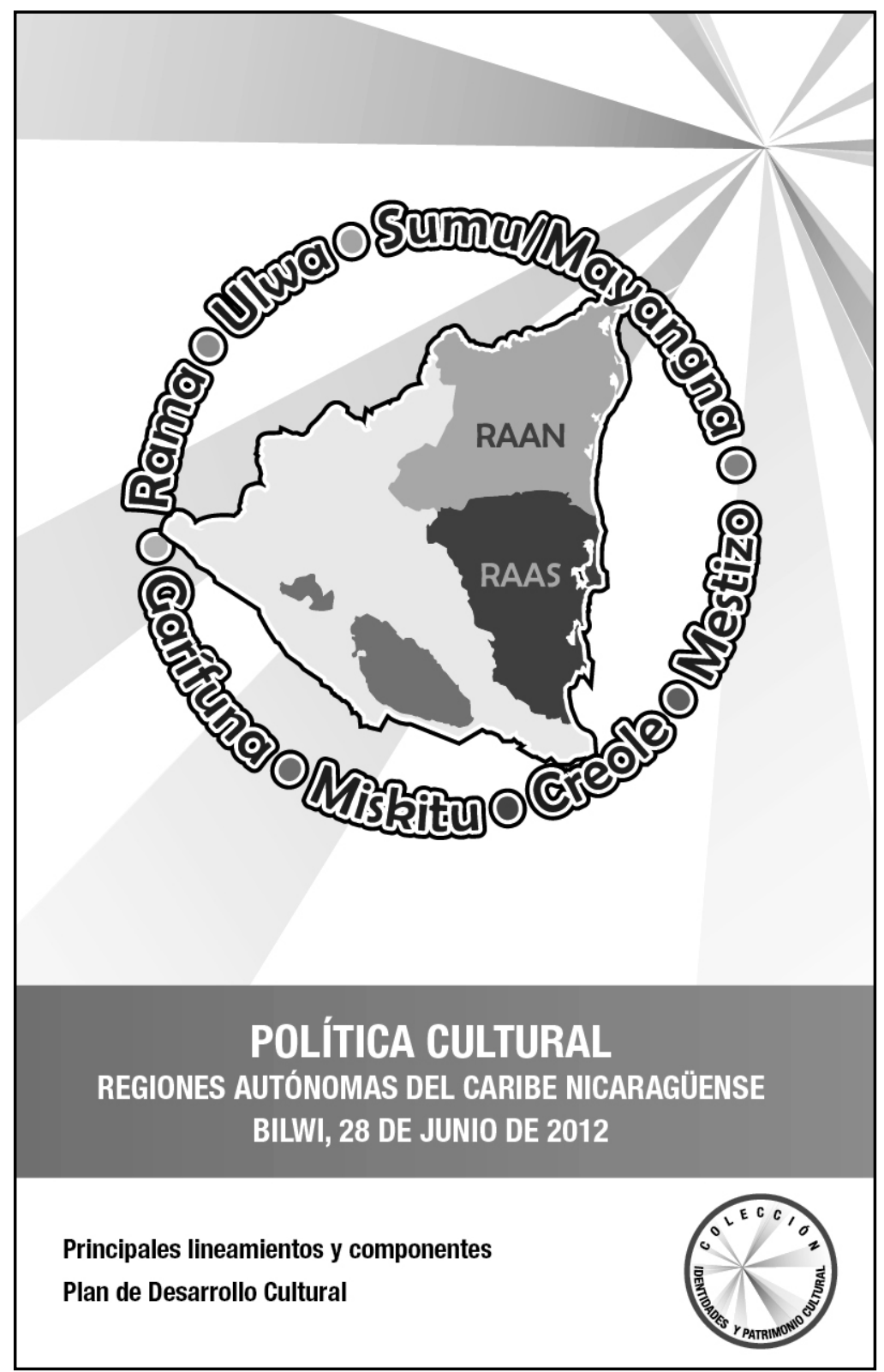
Autónomas de la Costa Atlántica de Nicaragua, (Ley No.28 de 1987) y su Reglamento, reafirman los derechos culturales de los pueblos indígenas y comunidades étnicas. El Estatuto, establece que a estas Regiones, les corresponde "promover el estudio fomento, desarrollo, preservación y difusión de las Culturas de la Comunidades de la Costa Atlántica de Nicaragua, así como su patrimonio histórico, artístico, lingüístico y cultural", además de promover la cultura nacional en las comunidades de la Costa Atlántica. La autonomía regional establece un marco multiétnico y pluricultural que es incluyente a pueblos indígenas, afrodescendientes y mestizos Costeños.

Los pueblos indígenas y comunidades afrodescendientes de la Costa Caribe de Nicaragua, tienen entre sus fortalezas contar con un marco legal que garantiza sus derechos fundamentales sobre el territorio, la propiedad colectiva de sus tierras y recursos, sus prácticas culturales, la lengua, los sistemas organizativos y de reconocimiento de las autoridades tradicionales comunitarias y territoriales, la disponibilidad de recursos humanos formados y universidades propias con un enfoque multicultural, plurilingüe, la presencia de recursos naturales diversos y una rica biodiversidad dentro de cada uno de los territorios,

Diagnóstico y Línea de Base Cultural 
así como expresiones culturales, artísticas que son recreadas y que visibilizan sus propias formas culturales.

\section{OBJETIVOS}

Son objetivos de la Política Cultural de las Regiones Autónomas los siguientes:

1. Contribuir al conocimiento, reconocimiento, rescate, conservación, restauración, revitalización y difusión de los valores, manifestaciones, prácticas y patrimonio culturales de los pueblos indígenas y comunidades étnicas de las regiones autónomas.

2. Brindar elementos que contribuyan al reconocimiento, ejercicio y respeto pleno de los derechos culturales de los pueblos indígenas y comunidades étnicas de las regiones autónomas.

3. Fortalecer la identidad y cohesión social de los pueblos indígenas y comunidades afrodescendientes de la Costa Caribe de Nicaragua.

4. Contribuir al desarrollo de la cultura nacional, desde una perspectiva multiétnica y pluri e intercultural, y al respeto y convivencia pacífica entre los pueblos indígenas, comunidades afrodescendientes y resto del país.

5. Fomentar una adecuada relación entre el patrimonio cultural de los pueblos indígenas y comunidades afrodescendientes y el potencial de desarrollo socioeconómico de las regiones autónomas del Caribe nicaragüense.

\section{PRINCIPIOS}

Los principios sobre los cuales se sustenta la Política Cultural de las Regiones Autónomas, son los siguientes:

1. Autonomía. El reconocimiento de que los pueblos indígenas, comunidades afrodescendientes y mestizos costeños, tienen la capacidad, responsabilidad, compromiso y empoderamiento necesarios para el ejercicio de sus derechos culturales, y para participar con efectividad en las decisiones e iniciativas culturales que sean adoptadas e impulsadas en el marco de la política cultural.

2. Integralidad. Aborda la política cultural, y su contenido, tomando en cuenta las dimensiones: Humanas (visiones, cosmovisiones, saberes, espiritualidades, valores, tradiciones, usos, costumbres, prácticas, formas de organización y normas de convivencia, y concepciones del desarrollo, y demás manifestaciones culturales), físicas (tierra, naturaleza, territorio, y recursos naturales), generacionales (respeto de la tradición indígena, afrodescendiente y mestiza costeña, basada en el reconocimiento ancestral, y en la transmisión intergeneracional de esos saberes, valores, tradiciones, usos, costumbres, prácticas y manifestaciones culturales).

3. Diversidad. El reconocimiento y respeto de las particularidades y diferencias culturales existentes entre los pueblos indígenas, comunidades afrodescendientes y mestizos costeños de las Regiones Autónomas.

4. Responsabilidad. El deber de los pueblos indígenas, comunidades afrodescendientes, y mestizos costeños, de sus líderes, autoridades comunales y territoriales, así como de las autoridades regionales, para impulsar acciones encaminadas al reconocimiento, respeto $\mathrm{y}$ salvaguarda de sus derechos culturales, incluyendo los derechos sobre sus bienes y patrimonio culturales.

5. Participación. La necesidad y responsabilidad de que en la definición e implementación de esas acciones participen plena y efectivamente, bajo el derechos del consentimiento previo, libre e informado, los pueblos indígenas, comunidades afrodescendientes y mestizos costeños

6. Transparencia. En el proceso de gestión, asignación y utilización de recursos destinados a la implementación de la política, estrategia y planes de desarrollo cultural, impulsando la participación activa y efectiva en la gestión directa de recursos por parte de los pueblos indígenas y comunidades afrodescendientes, y de sus autoridades en los ámbitos: regional, municipal, territorial y comunitario, y las instituciones nacionales.

7. Interculturalidad. El desarrollo de la política, estrategia y planes de desarrollo culturales, deben corresponderse y ser pertinentes con las particularidades culturales diferenciadas de cada uno de los pueblos indígenas y comunidades afrodescendientes de las regiones autónomas en sus propias cosmovisiones, conocimientos, valores, tradiciones, costumbres, espiritualidades, formas de organización y normas de convivencia, y concepciones del desarrollo, todas ellas resultantes de las relaciones sociales, de carácter pluricultural que se desarrollan en las dos regiones autónomas. 
8. Institucionalidad autonómica. La base para el desarrollo de la política, estrategia y planes de desarrollo culturales, deben elaborarse e implementarse en el marco del el respeto de la institucionalidad autonómica de las regiones autónomas, y de las normas de derecho positivo y consuetudinarias que la sustentan.

9. Pertinencia. El contenido de la política cultural, su estrategia de implementación, así como las acciones contempladas en los planes de desarrollo cultural deben corresponderse con las necesidades culturales y las oportunidades existentes para su reafirmación e identificación, de los pueblos indígenas, comunidades afrodescendientes y mestizos costeños de las Regiones Autónomas de la Costa Caribe de Nicaragua.

\section{LA POLÍTICA CULTURAL DE LAS REGIONES AUTÓNOMAS}

La cultura crea sentimientos de identidad común y de pertenencia, los cuales son elementos claves para la cohesión social ${ }^{2}$. Esta cohesión social es fundamental para el impulso del proceso de consolidación y profundización del régimen autonómico del cual gozan ambas Regiones. $\mathrm{Y}$, siendo que la Cultura es entendida por los pueblos indígenas, comunidades afrodescendientes y mestizos costeños, como:

Modos de vida, sistemas de valores, tradiciones y creencias de cada pueblo. Las culturas de los pueblos indígenas están basadas en sus conocimientos tradicionales, su visión del mundo y su espiritualidad. Éstos no son estáticos, al contrario, son dinámicos: producen innovaciones desde dentro, abarcan todos los aspectos de la vida comunitaria, se vinculan al manejo del entorno natural, son acumulativos y representan generaciones de experiencias, observación cuidadosa y experimentación constante, pero también usan y adaptan conocimientos externos

La política cultural de las Regiones Autónomas está constituida por el conjunto de lineamientos, estrategias, programas, proyectos, medidas y acciones, e institucionalidad, que en los ámbitos público y privado, enmarcados en el derecho internacional, la constitución política y marco jurídico particular que sustenta el
Régimen de Autonomía, rescaten, protejan, revitalicen, reafirmen, fortalezcan, consoliden y aseguren esos modos de vida, sistemas de valores, tradiciones, creencias, conocimientos tradicionales, modelos de desarrollo, visiones y espiritualidad de los pueblos indígenas, comunidades afrodescendientes y mestizos costeños en ambas regiones, así como el reconocimiento, respeto y ejercicio pleno de los derechos culturales inherentes; sustentados en los principios de diversidad cultural e interculturalidad, holismo e integralidad y universalidad.

\section{LÍNEAS DE LA POLÍTICA CULTURAL DE LAS REGIONES AUTÓNOMAS}

La Política Cultural de las Regiones Autónomas será implementada, atendiendo a las siguientes líneas y considerando las acciones previstas en cada una de ellas:

A. Preservación y fomento de los derechos, riqueza y diversidad culturales de los pueblos indígenas, comunidades afrodescendientes y mestizos costeños de las Regiones Autónomas

1. Fortalecer el ejercicio y respeto pleno de los derechos culturales de los pueblos indígenas, comunidades afrodescendientes y mestizos costeños de las regiones autónomas. Entre ellos, su derecho a: mantener y desarrollar su cosmovisión y visión holística, a que sean respetados sus valores y costumbres, al mantenimiento de sus tradiciones y formas de expresión cultural, a su libertad de creencias y prácticas culturales, a la libertad de creación intelectual, artística, técnica y científica.

2. Contribuir a la preservación de la diversidad, derechos, riqueza y patrimonio culturales, bases de la sociedad multiétnica de las regiones autónomas, de manera que se fortalezca su identidad, cohesión y sentido de pertenencia, elementos que forman parte de su capital social.

3. Impulsar programas y proyectos dirigidos a la protección, preservación, rescate, y revitalización de las tradiciones, expresiones, manifestaciones, prácticas, edificios, sitios, monumentos y centros culturales de los pueblos indígenas, comunidades

2 Cuenin, F. (2009). Patrimonio cultural y desarrollo socioeconómico: la recuperación de áreas centrales históricas (Nota Técnica). BID. New York. 
afrodescendientes y mestizos costeños de las regiones autónomas, incluyendo entre estos últimos, centros y sitios sagrados e históricos de gran significado para estos pueblos y comunidades.

\section{Promover que los medios masivos de comunicación de} las regiones autónomas y resto del país, contribuyan a difundir los valores y el patrimonio cultural de los pueblos indígenas, comunidades afrodescendientes y mestizos costeños de las regiones autónomas, incorporando en las actividades de promoción cultural a los sectores público y privado.

B. Fortalecimiento de la Cultura Autóctona de las Regiones Autónomas, articulando esfuerzos con la Política Cultural Nacional y sus Lineamientos.

5. Formular y ejecutar planes y programas culturales, para cada uno de los pueblos indígenas, comunidades afrodescendientes y mestizos costeños, en especial aquéllos que contribuyan al rescate y revitalización cultural, $\mathrm{y}$ al fortalecimiento de la pluriculturalidad e interculturalidad, considerando la diversidad cultural de estos pueblos y comunidades.

6. Incorporar la investigación científica y tecnológica, así como el conocimiento de las prácticas ancestrales en los ámbitos económicos, sociales y culturales, como parte sustancial del desenvolvimiento cultural, con énfasis en el estudio, prácticas, conocimientos y formas de innovación propios del entorno material y cultural de los pueblos indígenas, comunidades afrodescendientes y mestizos costeños de ambas regiones autónomas.

7. Promover la investigación, conservación, restauración y difusión del patrimonio cultural: tradicional, religioso, arqueológico, histórico, artístico y lingüístico así como los recursos naturales de los pueblos indígenas, comunidades afrodescendientes y mestizos costeños de las regiones autónomas.

8. Impulsar la creación, mantenimiento y desarrollo de centros culturales: museos, exposiciones artísticas, de historia natural, de arte, arqueología, historia y etnografía, ciencia y tecnología, y otros, estimulando a los gobiernos territoriales y comunales y al sector privado regional, a apoyar y desarrollar las iniciativas en esa dirección.
9. Impulsar el desarrollo de iniciativas culturales particulares, de carácter individual y colectivo, entre los pueblos indígenas, comunidades afrodescendientes y mestizos costeños de las regiones autónomas, favoreciendo el reconocimiento de Nicaragua como un país multicultural, multiétnico y plurilingüe.

10. Promover y difundir, en las Regiones Autónomas, el conocimiento de la cultura nacional, regional, caribeña y universal, sin discriminar su procedencia o credo, estimulando la producción y circulación de publicaciones, videos y otros medios de conservación y comunicación del conocimiento.

11. Contribuir a la implementación del principio de convivencia pacífica intercultural entre los pueblos indígenas, comunidades afrodescendientes y mestizos costeños y resto del país, favoreciendo el rescate , validación, promoción y defensa de los rasgos propios de nuestra identidad cultural, y promoviendo que las instancias de gestión cultural, en los ámbitos municipal, territorial, regional y nacional, interactúen con las instancias propias de nuestros.

C. Impulso y fortalecimiento del rol de la cultura y su contribución en la ejecución de planes y programas que favorezcan el desarrollo de las Regiones Autónomas y el "buen vivir" y el bien común de los pueblos indígenas, comunidades afrodescendientes y mestizos costeños.

12. Asociar las estrategias y programas culturales, de las regiones autónomas, con sus políticas, estrategias, planes, programas y proyectos de desarrollo económico y con aquéllos dirigidos al fortalecimiento y consolidación del proceso autonómico.

13. Preservar, impulsar y fortalecer, de forma adecuada la relación existente entre el patrimonio cultural de los pueblos indígenas, comunidades afrodescendientes y mestizos costeños y el potencial de desarrollo socioeconómico de las regiones autónomas, privilegiando el respeto a los derechos culturales de esos pueblos y comunidades.

14. Fomentar el desarrollo de las empresas culturales de las regiones autónomas, que favorezcan la producción de bienes y servicios culturales que reflejen los contenidos propios de la cultura de los pueblos indígenas, comunidades afrodescendientes y mestizos costeños de ambas regiones. 
D. Promoción e impulso de acciones encaminadas al respeto y protección de la propiedad intelectual y la certificación del origen de productos propios de los pueblos indígenas, comunidades afrodescendientes y mestizos costeños de las Regiones Autónomas.

15.Impulsar la creación de mecanismos jurídicos $y$ administrativos dirigidos al reconocimiento $y$ respeto de la propiedad intelectual, de los pueblos indígenas, comunidades afrodescendientes $y$ mestizos costeños de ambas regiones, sobre sus creaciones, invenciones, conocimientos tradicionales, formas de aprovechamiento ancestral de sus recursos naturales, y de la biodiversidad existente en los territorios que han habitado por miles de años.

16. Establecer un mecanismo específico, por parte del Consejo Regional Autónomo orientado al reconocimiento de la propiedad y la certificación del origen de productos provenientes de un determinado pueblos indígena, afrodescendientes o mestizo costeño.

\section{PRINCIPALES COMPONENTES DEL PLAN DE DESARROLLO CULTURAL}

Como hemos indicado, el proceso de devolución de resultados del diagnóstico e inventario cultural ha permitido identificar los recursos culturales que deben ser protegidos y promovidos por cada uno de los Pueblos Indígenas, Comunidades Afrodescendientes y mestizos costeños de las Regiones Autónomas de la Costa Caribe de Nicaragua, con el propósito de:

1. Garantizar su permanencia histórica en la memoria colectiva de los habitantes de las Regiones Autónomas.

2. Contribuir al fortalecimiento de la cultura e identidad de esos pueblos y comunidades.

Los componentes y acciones definidos, se sustentan en las líneas de la política propuestas y en las consideraciones efectuadas por cada uno de los pueblos indígenas, comunidades afrodescendientes y mestizos costeños. Y, están dirigidos a la protección y promoción de sus prácticas culturales y mantenimiento de sus estilos de vida, así como al impulso de iniciativas que visibilicen, articulen, mejoren, promuevan, consoliden e institucionalicen los rasgos particulares de la cultura de los Pueblos ${ }^{3}$ con vistas al fortalecimiento de su identidad como pueblos y comunidades diferenciadas.

También, en las debilidades de las que adolecen las Regiones Autónomas, las cuales incluyen aquéllas asociadas a la poca construcción de procesos e iniciativas institucionales que garanticen y promuevan las expresiones y prácticas culturales tradicionales, las limitadas capacidades en las estructuras de gestión comunitaria para el desarrollo y protección de su cultura y su patrimonio así como a la escasa asignación presupuestaria y transparencia en el manejo de los fondos de promoción cultural. A ello se agregan la ausencia de articulación de una política y estrategia regional que garantice la promoción y divulgación de expresiones culturales y artísticas y las limitadas capacidades de protección de los bienes culturales y arqueológicos y, de conocimientos y saberes tradicionales existentes en cada uno de los territorios de los pueblos indígenas y comunidades afrodescendientes, así como el desconocimiento de su valor real; la pérdida lingüística en algunos pueblos indígenas como el Rama, Panamahka, Ulwa, Twahka, Sumu-Mayangna y Garífuna, y la falta de divulgación de elementos culturales.

Entre las principales componentes y acciones propuestas están las siguientes:

\section{A. Protección de los recursos culturales individuales, colectivos, materiales y tradiciones culturales locales, empresas e infraestructuras culturales ${ }^{4}$.}

Protección de tradiciones culturales ${ }^{5}$, mediante:

i. Realización de estudios, que ayuden a recuperar y generar espacios de conocimiento que se van perdiendo, con participación plena y efectiva de los pueblos indígenas, comunidades afrodescencientes y mestizos costeños.

3 Informe final Devolución y Validación de los Informes de los Diagnósticos y Mapeos Culturales de los Pueblos Indígenas y Afro descendientes de la RAAN y RAAS.

${ }^{4}$ En el informe final de devolución y validación de los Informes de los Diagnósticos y Mapeos Culturales se ha identificado un total de 194 recursos culturales que deben ser protegidos y promovidos. $39.8 \%$ de estos recursos forman parte del patrimonio del Pueblo Mískitu, 24.5 \% al Mayangna, $13.3 \%$ al Garífuna, $8.7 \%$ al Rama, $4.6 \%$ al Ulvas. $9.2 \%$ de los recursos fueron propuestos por participantes en los talleres de Devolución en la URACCAN y la BICU, en los que según sus características deben de anexárseles al Pueblo Mískitu.

5 Cuentos, mitos, creencias, lenguas, historias, música. 
ii. Divulgación y transmisión educativa e informativa, la elaboración de diccionarios en las lenguas maternas.

iii. Implementación del Sistema Educativo Autonómico Regional (SEAR) en la parte de reconocimiento $\mathrm{y}$ aplicación del pensum académicos relacionado con expresiones culturales vinculados al patrimonio de los pueblos indígenas y afrodescendientes y mestizos.

iv. Aprovechamiento del marco legal que brinda el marco legal nacional y el Estatuto de la Autonomía para formular declaratorias del patrimonio cultural tradicional de las Regiones Autónomas, por parte de los Consejos Regionales.

v. Realización de asambleas comunitarias, encuentros e intercambio inter-culturales.

Protección de recursos materiales (Edificios y monumentos, grabados en piedra, escritos, documentos históricos, sitios turísticos, espacios naturales, bosques y sitios sagrados).

vi. Establecimiento de acuerdos, convenios y alianzas que garanticen la conservación futura, de los recursos materiales, y la ley de protección del patrimonio cultural de la nación.

vii. Definición e implementación de normas y regulaciones que limiten la presencia de personas ajenas que puedan dañarlos y usurparlos.

viii. Orientar programas de financiamiento y fortalecimiento de capacidades que garanticen la operatividad y sostenibilidad futura y a la generación de empleos para el caso de las empresas turísticas, y los sitios de interés y potencial turístico.

ix. Reforestación de áreas degradadas para su recuperación, en las cercanías y alrededor de ríos, riachuelos y criques. Así como de bosques intervenidos para aprovechamiento de especies arbóreas con fines de autoconstrucción y comercialización ${ }^{6}$.

x. Establecer normas comunitarias para la protección de humedales e inclusive establecer vedas que garanticen su recuperación.

xi. Establecer rutas y mecanismos para promover y fomentar el turismo, en los Cayos e Islas, como iniciativa a fin de generar fuentes alternativas de ingresos económicos para los Pueblos Indígenas, Comunidades Afrodescendientes y mestizos costeños.

xii. Facilitar mecanismos de créditos en el marco de la propiedad colectiva.

xiii. Aprovechamiento del marco legal que brinda el Estatuto de la Autonomía, para formular declaratorias del patrimonio cultural material de las Regiones Autónomas, por parte de los Consejos Regionales.

1. Protección de infraestructuras culturales (casas, cementerios).

i. Implementación de programas orientados a su rehabilitación y embellecimiento, conservación y protección de casas con arquitectura local, templos, cementerios, monumentos.

\section{B. Promoción de los Recursos Culturales de los Pueblos Indígenas y Comunidades Afrodescendientes de la Costa Caribe.}

2. Establecimiento de mecanismos de promoción que contribuyan a garantizar la transmisión generacional de los conocimientos culturales que disponen los poseedores tradicionales, a través de:

i. Promoción de la participación plena y efectiva y bajo la consulta y consentimiento previo, libre e informado, a través de asambleas comunitarias, intercambios, consultas, cabildos y otros.

ii. Desarrollo de procesos de enseñanza, charlas, elaboración de libros en versiones populares que faciliten la asimilación por parte de las nuevas generaciones.

iii. Documentación y transmisión de elaboración de textos de enseñanza en lenguas autóctonas de las Regiones Autónomas.

iv. Realización de grabaciones y videos para ser trasmitido en programas radiales y televisivos, en iglesias y centros de aprendizaje.

${ }^{6}$ Considerando la especial relación que mantienen los Pueblos Indígenas con la Naturaleza, en la que se conjuga lo tradicional con lo espiritual y sagrado. 
v. Realización de encuentros comunales, ínter comunales e inter regionales que faciliten la divulgación y apropiación cultural, de las danzas, cantos, literatura, artesanía, deportes y otros.

3. Promover la cultura en cada uno de los Pueblos Indígenas, Afrodescendientes y mestizos costeños. por medio de espacios radiales, televisivos, ferias, talleres, teatros populares, danzas, bailes y cantos.

4. Dar a conocer estudios y documentos realizados sobre la cultura, recursos naturales y paisajes existentes de nuestros pueblos indígenas, comunidades afrodescendientes y mestizos costeños en las Regiones Autónomas, como elemento de transmisión y apropiación cultural.

5. Desarrollar mecanismos de transmisión sistemática de los conocimientos existentes en los portadores del conocimiento tradicional (ancianos, ancianas,) sobre medicina tradicional, prácticas espirituales, agrícolas, estrategias de navegación, caza y pesca, para garantizar la continuidad de estos saberes como elementos contributivos a la identidad de los pueblos.

6. Promover acciones que vincule la producción artesanal comunitaria como medio para impulsar las economías locales y la promoción de expresiones artísticas propias.

7. Impulsar a través de las universidades regionales, centros de investigación o instituciones del Estado, la planificación y el desarrollo de investigaciones de expresiones culturales, en particular en sitios y lugares inexplorados y generar conocimientos sobre el pasado de estos pueblos.

8. Promover el desarrollo de estudios e investigaciones para el rescate de la memoria histórica y la registro de sus mecanismos de socialización.

9. Promover el fortalecimiento de los procesos de rescate y revitalización lingüística de cada pueblo, mediante el desarrollo de investigaciones, documentales, elaboración de libros y folletos, grabaciones radiales y televisivas, encuentros, talleres, seminarios, y docencia formal e informal.

\section{Creación y fortalecimiento de capacidades locales para la promoción y divulgación de las prácticas y expresiones culturales existentes.}

10. Fomento de programas de formación, investigaciones y publicaciones, y ferias de intercambio y socialización cultural, y dar seguimiento a un programa de formación en gestión cultural dirigido a fortalecer las capacidades locales en esa materia.

11. Implementación de los planes de desarrollo culturales de los pueblos, que respondan a nuestras propias realidades y condiciones de vida $y$, que orientan y dan pautas al quehacer institucional para la recuperación, conservación y sostenibilidad de sus expresiones y patrimonio cultural.

12. Destinar una asignación presupuestaria para el adecuado desempeño de funciones, responsabilidades y obligaciones de las instituciones vinculadas al fortalecimiento de las prácticas culturales y gestionara recursos externos.

13. Asegurar una distribución efectiva, justa y equitativa, de los fondos culturales y crear estas capacidades en los municipios, territorios y comunidades locales.

14. A nivel educativo fomentar y poner en prácticas aprendizajes desde lo propio, por cada uno de las lenguas, documentar y trasladar a versión popular los textos de enseñanza que garanticen la apropiación por los jóvenes pero también de los adultos y personas mayores.

\section{A. Sensibilización para la Conservación de los Recursos Culturales}

1. Realizar campaña de sensibilización para la conservación de los recursos culturales; materiales y expresiones culturales través de todos los medios de comunicación.

2. Efectuar capacitaciones a los diferentes actores comunitarios e institucionales como mecanismo de apropiación de prácticas culturales de cada pueblo y que los ayuden a trabajar con estas comunidades y aportar en la construcción colectiva del desarrollo. 
3. Desarrollar intercambio de experiencias culturales entre los pueblos, como un eslabón de intercambio, recreación y de compartir conocimientos.

4. Desarrollar estrategias de investigaciones culturales por pueblo, con la finalidad de identificar y tipificar los recursos materiales, y expresiones culturales y divulgar los hallazgos interna y externamente.

\section{LA ORGANIZACIÓN PARA LA IMPLEMENTACIÓN DE LA POLÍTICA}

La necesidad de contar con una institucionalidad encargada de impulsar el proceso de implementación de la política cultural, así como de los planes de desarrollo cultural que se le deriven, supondría:

1. Que los Consejos Regionales de las Regiones Autónomas, convocarán a las autoridades municipales, territoriales, comunales y agentes culturales así como también las instituciones nacionales vinculadas a la implementación de estas políticas culturales para consultar la definición de e implementación de los principales lineamientos de la política cultural. Cualquiera definición o reforma que nazca de estas consultas serán aprobadas en el plenario del Consejo Regional Autónomo.

2. Las disposiciones administrativas y resoluciones de los Consejos Regionales Autónomos del Atlántico Norte y Sur, serán implementadas por las Secretarías de Cultura de los Gobiernos Regionales, unidades de cultura de las municipalidades, los gobiernos territoriales, comunal y las instituciones nacionales existentes en las Regiones Autónomas. Las Secretarías de Cultura estarán organizadas por diversas áreas funcionales: Gestión Cultural, Investigación y Rescate Cultural, Revitalización Cultural, Patrimonio Cultural, entre otras.

\section{PASOS PARA IMPLEMENTAR LA POLÍTICA CULTURAL DE LAS REGIONES AUTÓNOMAS}

De forma general se considera el desarrollo de los siguientes pasos, a fin de implementar la Política Cultural:

1. Definición de principales programas, por pueblo indígena, comunidades afrodescendientes y mestizos costeños, de acuerdo a las principales líneas y acciones de la política cultural.

2. Elaboración y aprobación de resolución de los Consejos Regionales, mediante la cual se establezcan los mecanismos específicos para proteger la propiedad intelectual colectiva y la certificación de origen de los conocimientos colectivos, productos y bienes culturales de los pueblos indígenas y comunidades étnicas.

3. Definición, estructural y funcional, de la institucionalidad encargada de poner en práctica la política cultural de las regiones autónomas y sus lineamientos. 
Aprobado en la ciudad de Bilwi, Región Autónoma Atlántico Norte, a los veintiocho días del mes de junio del año dos mil doce, suscriben:
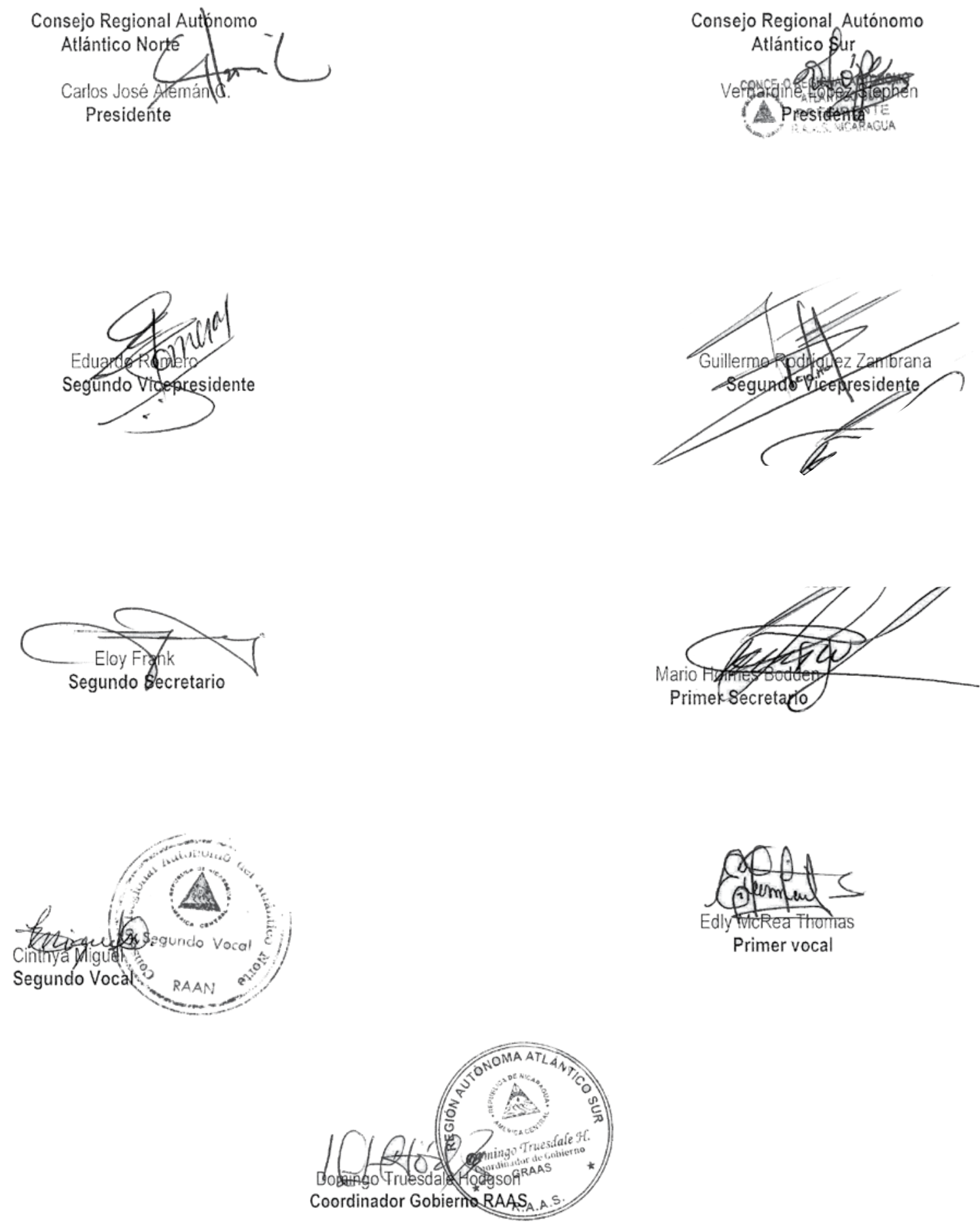